\title{
No-Boundary Proposal as a Path Integral with Robin Boundary Conditions
}

\author{
Alice Di Tucci and Jean-Luc Lehners \\ Max-Planck-Institute for Gravitational Physics (Albert-Einstein-Institute), 14476 Potsdam, Germany
}

(Received 18 March 2019; published 23 May 2019)

\begin{abstract}
Realizing the no-boundary proposal of Hartle and Hawking as a consistent gravitational path integral has been a long-standing puzzle. In particular, it was demonstrated by Feldbrugge, Lehners, and Turok that the sum over all universes starting from a zero size results in an unstable saddle point geometry. Here we show that, in the context of gravity with a positive cosmological constant, path integrals with a specific family of Robin boundary conditions overcome this problem. These path integrals are manifestly convergent and are approximated by stable Hartle-Hawking saddle point geometries. The price to pay is that the off-shell geometries do not start at a zero size. The Robin boundary conditions may be interpreted as an initial state with Euclidean momentum, with the quantum uncertainty shared between the initial size and momentum.
\end{abstract}

DOI: 10.1103/PhysRevLett.122.201302

If the quantum theory is universal, and there currently is no reason to think otherwise, then the Universe should be describable by a quantum state just like any other system. While its quantum properties might be hidden today, they may well have played a crucial role in an early phase of its evolution. An intriguing idea in this context is that a finite universe might have made its appearance out of nothing due to something akin of a quantum tunneling effect. In fact, this idea has a long history dating back all the way to Lemaitre [1]. Quantum cosmology should then be able to describe such a process. The most concrete formulations of this idea go under the names of "no-boundary proposal" [2] and "tunneling proposal" [3]. The path integral formulation of these proposals has recently been analyzed in the context of Lorentzian quantum cosmology [4-8]. There, a transition amplitude from a geometry of zero size, i.e., from "nothing," to a finite one was evaluated. It was shown that the two proposals are, in fact, identical in this formulation and that the path integral gives a result analogous to the tunneling amplitude described in Ref. [3]. Perturbations around the background geometry, however, turn out to be unstable, meaning that larger and larger deviations from homogeneity and isotropy are favored quantum mechanically. This led to the conclusion that neither the noboundary nor the tunneling proposal can be appropriate descriptions of the initial conditions of our Universe.

In follow-up works, different implementations of the proposals were proposed in order to avoid this negative conclusion. One attempt by Dorronsoro et al. was to

Published by the American Physical Society under the terms of the Creative Commons Attribution 4.0 International license. Further distribution of this work must maintain attribution to the author(s) and the published article's title, journal citation, and DOI. Funded by SCOAP ${ }^{3}$. consider intrinsically complex contours of integration for the lapse function [9], also in conjunction with a specification of the initial momentum [10]. However, these modifications either still included unstable perturbations [6] or led to inconsistencies [11]. Another approach by Vilenkin and Yamada (in the context of the tunneling proposal) was to modify the boundary conditions for the perturbations to be of the Robin type [12,13]. A final proposition by Halliwell, Hartle, and Hertog was to simply abandon the path integral and concentrate on solutions of the Wheeler-DeWitt equation with desirable properties [14] (see also [15]). However, the path integral neatly captures quantum interference, and focusing on solutions that cannot be described via a path integral may thus not correctly reproduce central quantum effects.

In this Letter, we will combine several of the ideas mentioned above, using Robin boundary conditions in order to impose a condition on a linear combination of the initial size and momentum of the Universe. For a family of such conditions, we find that the path integral can be approximated solely by the stable no-boundary saddle points, thus avoiding instabilities and representing a consistent definition of the no-boundary proposal.

But first, it seems appropriate to briefly review the negative result that we are trying to overcome. We will work in minisuperspace, where the geometries considered are of the form [16] $d s^{2}=-\left(N^{2} / q\right) d t^{2}+q d \Omega_{3}^{2}$, with $d \Omega_{3}^{2}$ a three-sphere of volume $2 \pi^{2}$. The function $q(t)$ is the squared scale factor, while $N(t)$ is the lapse function. The propagator for the no-boundary proposal $G\left[q_{1}, 0\right]$ describes a transition from a spatial 3-geometry of zero size, $q_{0}=0$, to a later one of size $q_{1}$. The propagator can be evaluated as a path integral over 4-geometries with a weighting $e^{i S}$ given by the Einstein-Hilbert action with a cosmological constant $\Lambda=3 H^{2}$. The steps needed to define the gravitational path 
integral can be found in Refs. [17,18], with the final result (in constant lapse gauge $\dot{N}=0$ )

$$
G\left[q_{1}, 0\right]=\int_{0^{+}}^{\infty} d N \int_{q(t=0)=0}^{q(t=1)=q_{1}} \delta q e^{i S / \hbar},
$$

where the action takes the form (with $8 \pi G=1$ )

$$
\begin{aligned}
S & =\int d^{4} x \sqrt{-g}\left(\frac{R}{2}-\Lambda\right) \\
& =6 \pi^{2} \int_{0}^{1} d t\left[-\frac{\dot{q}^{2}}{4 N}+N\left(1-H^{2} q\right)\right] .
\end{aligned}
$$

The integration domain for the lapse is $N \in\left(0^{+}, \infty\right)$, ensuring that the geometries in the sum have a Lorentzian signature. This makes the path integral a propagator in the sense that it solves the inhomogeneous Wheeler-DeWitt equation $\hat{\mathcal{H}} G\left[q_{1}, 0\right]=-i \delta\left(q_{1}\right)$, where $\hat{\mathcal{H}}$ is the quantum Hamiltonian.

It has been shown in Ref. [4] that the result of the various integrations to leading order in $\hbar$ is

$$
G\left[q_{1}, 0\right]=e^{-\left(4 \pi^{2} / H^{2} \hbar\right)-i\left(4 \pi^{2} H / \hbar\right)\left[q_{1}-\left(1 / H^{2}\right)\right]^{3 / 2}},
$$

where we assumed that $q_{1}>1 / H^{2}$. The negative weighting $-\left(4 \pi^{2} / \hbar H^{2}\right)$ is characteristic of the tunneling proposal [3], whereas the conjectured no-boundary result is [2]

$$
\Psi\left(q_{1}\right)=e^{+\left(4 \pi^{2} / H^{2} \hbar\right)} \cos \left[\frac{4 \pi^{2} H}{\hbar}\left(q_{1}-\frac{1}{H^{2}}\right)^{3 / 2}\right] .
$$

The difference between the two proposals may be elucidated by considering the saddle points of the full path integral (1). There are four saddle points, with geometries given by $q(t)=H^{2} N^{2} t(t-1)+q_{1} t$, with the lapse taking the values $N_{c_{1}, c_{2}}=\left(c_{1} / H^{2}\right)\left(\sqrt{H^{2} q_{1}-1}+c_{2} i\right)$ for $c_{1}, c_{2} \in\{-1,1\}$. Their action is

$$
S\left(N_{c_{1}, c_{2}}\right)=c_{1} \frac{4 \pi^{2}}{H^{2}}\left[c_{2} i-\left(H^{2} q_{1}-1\right)^{3 / 2}\right] .
$$

From this expression we see that the propagator (2) is given by the contribution of the saddle point with $c_{1}=1$ and $c_{2}=1$, in the upper right quadrant of the complex $N$ plane. It is, in fact, possible to show, applying the PicardLefschetz theory, that the integral along the positive real $N$ line is equivalent to the integral along the steepest descent path ("thimble") running through this saddle point alone; see Fig. 1. This saddle point is, however, unstable [5,19]. Thus, the result (2), though mathematically correct, cannot describe our Universe on physical grounds.

The no-boundary result (3) instead would have been obtained by considering the contribution of the two saddle points in the lower half complex $N$ plane, with $c_{1}=1$,

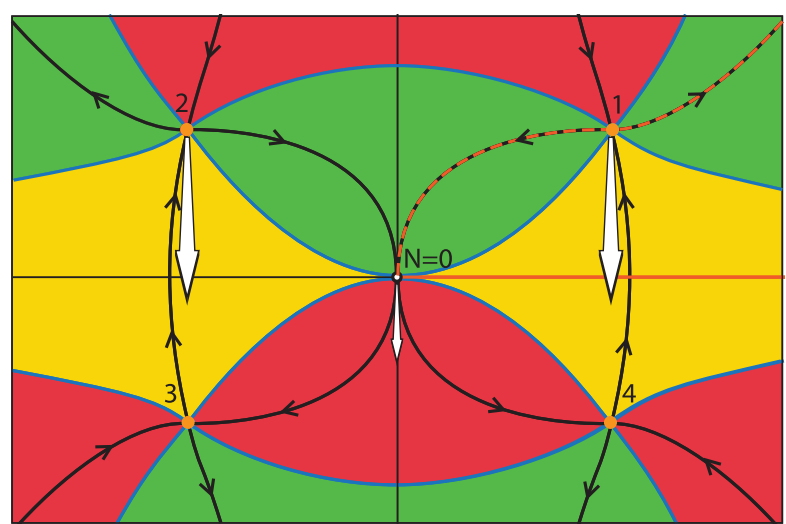

FIG. 1. The minisuperspace path integral contains four saddle points (orange dots) in the plane of the complex lapse $N$. Steepest descent or ascent lines of the magnitude of the action are drawn as black lines, with the arrows indicating descent. Asymptotic regions of convergence are shown in green, and divergent ones are red. For the path integral with Dirichlet boundary conditions, the defining contour of a positive real lapse (orange line) can be deformed to the (orange dashed) thimble flowing through saddle point 1 only. With Robin boundary conditions, saddle points 1 and 2 and the singularity of the action at $N=0$ move. Shown here is the direction of motion for negative imaginary $\beta$.

$c_{2}=-1$ and $c_{1}=-1, c_{2}=1$. There is, however, no convergent contour which can be deformed into a steepest descent path running through solely these two saddle points [6]. In this sense, the wave function (3) is not the saddle point approximation of the no-boundary wave function for any Lorentzian path integral with these boundary conditions. With different boundary conditions, the situation may change, as we will now discuss.

Let us consider augmenting the action with a Robin boundary term at the initial surface:

$$
S_{\mathrm{tot}}=S+\alpha q_{0}+\frac{q_{0}^{2}}{2 \beta} .
$$

The variation of the full action is now

$$
\delta S_{\text {tot }}=2 \pi^{2} \int_{0}^{1} d t\left(\frac{3 \ddot{q}}{2 N}-3 N H^{2}\right) \delta q-\frac{3 \pi^{2}}{N} \dot{q}_{1} \delta q_{1}+\mathcal{B} \delta q_{0}
$$

Thus, we can see that the variational principle is well defined if we impose $\delta q_{1}=0$ and

$$
\mathcal{B} \equiv \frac{3 \pi^{2}}{N} \dot{q}_{0}+\alpha+\frac{q_{0}}{\beta}=0 .
$$

In other words, we fix the field value at $t=1$ to be $q(t=1)=q_{1}$ corresponding to a Dirichlet boundary condition, while at $t=0$ we impose a condition on the linear combination of $q_{0}$ and $\dot{q}_{0}$. With these boundary conditions, the solution of the equation of motion reads 


$$
\begin{aligned}
q(t)= & H^{2} N^{2} t^{2}-\frac{\left(\alpha \beta+q_{1}-H^{2} N^{2}\right)}{3 \pi^{2} \beta-N} N t \\
& +\frac{3 \pi^{2}\left(q_{1}-H^{2} N^{2}\right)+N \alpha}{3 \pi^{2} \beta-N} \beta .
\end{aligned}
$$

Plugging this solution back into the action, we find that the saddle points are

$$
N_{s}=3 \pi^{2} \beta+c_{1} \frac{\sqrt{H^{2} q_{1}-1}}{H^{2}}+c_{1} c_{2} \frac{\sqrt{9 \pi^{4} \beta H^{4}-\alpha \beta H^{2}-1}}{H^{2}} .
$$

A crucial requirement in order to obtain an implementation of the no-boundary idea is that, at the saddle point, the geometry should be of Hawking type, and, in particular, it should start at a zero size. From (4), one can see that the initial size $\bar{q}_{0}$ vanishes at one (or more) of the saddle points if $\alpha= \pm 6 \pi^{2} i$ or $\beta=0$. For $\alpha=+6 \pi^{2} i$, this occurs at the "tunneling" saddle points $c_{1}=c_{2}=1$ and $c_{1}=c_{2}=-1$. These are, however, unstable, so that in the following we will consider $\alpha=-6 \pi^{2} i$ (we will discuss the meaning of $\beta$ momentarily). This gives $\bar{q}_{0}=0$ for

$$
N_{3,4}=\frac{-i}{H^{2}} \mp \frac{\sqrt{H^{2} q_{1}-1}}{H^{2}},
$$

which are precisely the Hartle-Hawking saddle points. The other two saddle points are now located at

$$
N_{1,2}=\frac{i}{H^{2}} \pm \frac{\sqrt{H^{2} q_{1}-1}}{H^{2}}+6 \pi^{2} \beta,
$$

and their initial size is $\bar{q}_{0}=12 \pi^{2} \beta\left(i+3 \pi^{2} \beta H^{2}\right)$. Therefore, requiring that at least one of the saddle point geometries starts out at a zero size corresponds to a specific value of $\alpha$ but leaves $\beta$ free. The value of $\beta$, in fact, determines which saddle point(s) are relevant to the path integral.

For $\beta=0$, the relevant saddle point is $N_{1}$. In this limit, the Robin boundary condition reduces to the Dirichlet boundary value $q_{0}=0$. For nonzero $\beta$, the saddle points in the upper half plane move, and the singularity in the action, which originally resides at $N^{\star}=0$, is also shifted to $N^{\star}=3 \pi^{2} \beta$. Importantly, the lower saddle points (numbers 3 and 4 in Fig. 1), which correspond to the desired HartleHawking geometries, stay put.

Our strategy will be to define the path integral on a thimble, for the simple reason that it is then manifestly convergent. This has the important consequence that the partial integrations over the scale factor, the lapse and any other fields that might be present, can be performed in any desired order without changing the end result (i.e., Fubini's theorem applies). At vanishing $\beta$, our defining integration contour is simply the Lorentzian one, along real positive values of $N$. This can then be deformed, using the Picard-Lefschetz theory, to the thimble passing through the unstable saddle point 1 , as shown in Fig. 1. As $\beta$ is turned on, the singularity of the integral at $N=0$ shifts to $N^{\star}$, and thus, in order to maintain an invariant definition, we will consider as our integration contour the thimble(s) emanating from $N^{\star}$.

But which value should $\beta$ take? Roughly speaking, the unstable saddle points remain relevant until $\beta$ is large enough in magnitude so that they have moved "out of the way." For real negative $\beta$, this approximately means that they have to move further to the left than $N^{\star}$, which marks the origin of the integration contour. But, since the real part of the saddle points depends on $q_{1}$, this is nonsensical from a physical point of view, as the initial conditions would have to keep being readjusted as the Universe keeps expanding. On the other hand, in the imaginary $N$ direction, the unstable point [with an original location at $\left.\operatorname{Im}\left(N_{1}\right)=+i / H^{2}\right]$ has to move only beyond the stable one at $\operatorname{Im}\left(N_{4}\right)=-i / H^{2}$. This condition is independent of the final size of the Universe, and thus we will consider only imaginary $\beta$. The minimal magnitude of $\beta$ is determined precisely by the condition that the unstable saddle point move below the stable one:

$$
|\beta|>\beta_{\min }=\frac{1}{3 \pi^{2} H^{2}} .
$$

For $\beta$ being negative imaginary and of a magnitude larger than this minimal value, the Hartle-Hawking saddle point becomes the only relevant one-see Fig. 2 for an illustration. Thus, we have successfully isolated the HartleHawking saddle point, leading to the propagator

$$
G\left[q_{1}, 0\right]_{\text {Robin }}=e^{+\left(4 \pi^{2} / H^{2} \hbar\right)-i\left(4 \pi^{2} H / \hbar\right)\left[q_{1}-\left(1 / H^{2}\right)\right]^{3 / 2}} .
$$

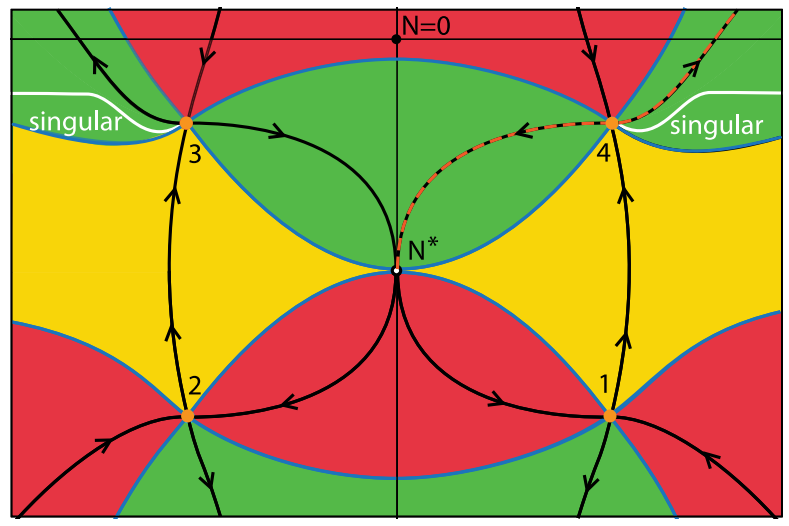

FIG. 2. For sufficiently large negative imaginary $\beta$, the unstable saddle point 1 moves below the Hartle-Hawking saddle point 4 . The thimble emanating from the singularity of the action at $N^{\star}$ now passes through only this stable saddle point, and the problem with instabilities is avoided. In white, we indicate the locus of geometries that contain a singularity and which we require the thimble to avoid. 


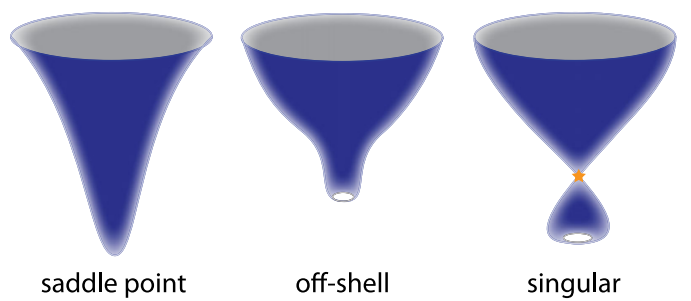

FIG. 3. On the left, we have the smooth saddle point geometry of Hartle-Hawking type. By contrast, with Robin boundary conditions a typical off-shell geometry will not start at a zero size (middle). Some off-shell geometries contain a recollapse to a zero size, and it is these geometries that we would like to avoid summing over (right).

In fact, we also have the possibility of including both thimbles emanating from $N^{\star}$, passing through saddles 3 and 4 in Fig. 2. If we define the integration contour to run over all real values of $N$, from minus infinity to plus infinity, the result will be the real no-boundary wave function (3) proposed by Hartle and Hawking. Moreover, in the latter case, the wave function will satisfy the homogeneous Wheeler-DeWitt equation $\hat{\mathcal{H}} \Psi=0$ (cf. the Appendix of Ref. [4]).

The Robin boundary condition implies a relationship between the initial size and the initial momentum of the geometries summed over in the path integral. It does, however, not necessarily avoid the appearance of singularities, in the sense that it may be possible that some of the off-shell geometries contain a region where the size of the Universe passes through zero; see Fig. 3. We would like to avoid summing over such geometries, as they are (infinitely) sensitive to the addition of higher curvature terms. This will imply an upper bound on $|\beta|$.

Thus, we must analyze the locus of geometries containing a region of zero volume. We start by noting that the imaginary part of the scale factor vanishes at

$$
\tau=-\frac{3 \pi^{2} i \beta}{H^{2} m} \frac{\left[-q_{1}+6 \pi^{2} i \beta+H^{2}\left(n^{2}+m^{2}+6 \pi^{2} i \beta m\right)\right]}{\left[n^{2}+\left(m+3 \pi^{2} i \beta\right)^{2}\right]},
$$

where we have split the lapse into real and imaginary parts as $N=n+i m$. We must then determine if the real part of the scale factor (4) may simultaneously vanish, with $0 \leq \tau \leq 1$. There is no concise analytic expression for this function. At large magnitudes of the lapse, this singular curve quickly becomes horizontal; see Fig. 2 for a sketch. Therefore, the relevant question is whether near the saddle point the thimble, which asymptotically runs off to infinity at an angle of $\pi / 6$ [4], can avoid crossing the singular curve. Thus, at $N_{4}$ we want the angle $\theta$ of the thimble to be larger than the angle $\varphi$ of the singular curve. This latter angle is given by

$$
\tan (\varphi)=\frac{\left(-2+3 \pi^{2} i \beta H^{2}\right) \sqrt{H^{2} q_{1}-1}}{\left(q_{1}+3 \pi^{2} i \beta\right) H^{2}-4} .
$$

Meanwhile, the angle $\theta$ is found to be

$$
\tan (\theta)=\frac{-1+3 \pi^{2} i \beta H^{2}}{\sqrt{H^{2} q_{1}-1}+H \sqrt{q_{1}-6 \pi^{2} i \beta-9 \pi^{4} H^{2} \beta^{2}}},
$$

where we have assumed that $|\beta|>\beta_{\text {min }}$. The condition $\theta>\varphi$ is then satisfied for negative imaginary $\beta$ with

$$
|\beta|<\beta_{\max }=\frac{1}{3 \pi^{2} H^{2}} \frac{3 H^{2} q_{1}-4}{H^{2} q_{1}-2} \approx \frac{1}{\pi^{2} H^{2}},
$$

where we assumed $q_{1}>\left(2 / H^{2}\right)$. Thus, for negative imaginary $\beta$ with a magnitude between $\beta_{\min }$ and $\beta_{\max }$, the path integral is well defined and contains only the Hartle-Hawking saddle point(s).

Let us now explicitly verify that the perturbations are indeed suppressed. In our model, we have only gravitational wave perturbations to deal with. For a single mode with fixed polarization, the action at quadratic order is given by (see, e.g., $[5,6])$

$$
S^{(2)}=\pi^{2} \int_{0}^{1} d t N\left[q^{2} \frac{\dot{\phi}^{2}}{N^{2}}-l(l+2) \phi^{2}\right]
$$

where $\phi$ denotes the magnitude of the perturbation, which has been expanded in spherical harmonics with $l \geq 2$ being the principal quantum number. The extension to a sum over all modes is straightforward. The equation of motion for $\phi$ is, thus,

$$
\ddot{\phi}+2 \frac{\dot{q}}{q} \dot{\phi}+\frac{N^{2}}{q^{2}} l(l+2) \phi=0 .
$$

In order to solve it, it is convenient to rewrite the scale factor as $q(t)=H^{2} N^{2}(t-\gamma)(t-\delta)$, where $\gamma$ and $\delta$ can be read off from (4). Then we find that two linearly independent solutions for $\phi(t)$ are $f(t) / \sqrt{q}$ and $g(t) / \sqrt{q}$ with

$$
f(t), g(t)=\left[\frac{t-\delta}{t-\gamma}\right]^{ \pm(\mu / 2)}[(1 \mp \mu)(\gamma-\delta)+2(t-\gamma)]
$$

and $\mu^{2}=1-4 l(l+2) /\left[(\gamma-\delta)^{2} N^{2} H^{4}\right]$. At the saddle point, the parameters reduce to $\mu\left(N_{4}\right)=(l+1), \gamma\left(N_{4}\right)=0$, and $\delta\left(N_{4}\right)=-2 i /\left(\sqrt{H^{2} q_{1}-1}-i\right)$. It is straightforward to check that this implies that the mode $f$ blows up at $t=0$, whereas $g /\left.\sqrt{q}\right|_{t=0}=0$. Thus, regularity selects the mode $g$, whose action at the saddle point is given by

$$
S^{(2)}=i \phi_{1}^{2} \frac{l(l+1)(l+2)}{2 H^{2}}-\phi_{1}^{2} \frac{l(l+2) \sqrt{q_{1}}}{2 H}+\mathcal{O}\left(\frac{1}{\sqrt{q_{1}}}\right),
$$


where $\phi_{1}$ is the final (real) magnitude of the perturbation. The resulting amplitude $e^{i S^{(2)} / \hbar}$ describes Gaussian distributed perturbations with a scale-invariant spectrum. We conclude, therefore, that the model is stable against small deviations, since large values of $\phi_{1}$ are suppressed.

More generally, we can specify Robin boundary conditions for the perturbations. The full action for the perturbations is then $S_{\mathrm{tot}}^{(2)}=S^{(2)}+\alpha_{\phi} \phi_{0}+\left(\phi_{0}^{2} / 2 \beta_{\phi}\right)$, which leads to $\delta \phi_{1}=0$ and

$$
\delta \phi_{0}\left[-\frac{2 \pi^{2} q_{0}^{2} \dot{\phi}_{0}}{N^{2}}+\alpha_{\phi}+\frac{\phi_{0}}{\beta_{\phi}}\right]=0 .
$$

Any Robin boundary condition with $\alpha_{\phi}=0$ and arbitrary $\beta_{\phi}$ will then retain the stable mode at the saddle point, while also specifying initial conditions for the perturbations off shell. Since we are only summing over nonsingular geometries off shell, for sufficiently small perturbation amplitudes the approximation of the linear perturbation theory around the background will be justified, as the perturbations encounter no divergence anywhere. Thus, the inclusion of perturbations will leave the path integral well defined and stable.

As already pointed out in the case of perturbations by Vilenkin and Yamada [12,13], the Robin boundary condition can also be implemented as a Gaussian integral:

$$
\int d N d q e^{i S / \hbar} \int d q_{0} e^{i \alpha q_{0} / \hbar-\left(q_{0}^{2} / 2|\beta|\right)},
$$

where we must include an integration over the initial scale factor $q_{0}$. Owing to the fact that $\beta$ has to be negative imaginary, this may then also be interpreted as an initial coherent state, albeit one with a Euclidean momentum $\alpha$. The presence of a Euclidean momentum not only implements the idea of closing the geometry off in Euclidean time, but it also adds a positive weighting to the associated geometries. In this way, we can obtain a final result with an enhanced weighting $e^{+4 \pi^{2} /\left(\hbar H^{2}\right)}$, which in the implementation with Dirichlet boundary conditions was simply impossible [6]. Note that in this context $\sqrt{|\beta|}$ takes on the role of the uncertainty in the initial size $q_{0}$. And because we have a coherent state, the uncertainty in the initial momentum is then simply its inverse:

$$
\Delta q_{0}=\sqrt{|\beta|} \sim \frac{1}{H}, \quad \Delta p_{0}=\frac{\hbar}{\sqrt{|\beta|}} \sim \hbar H
$$

Thus, we see that, in order to have a well-defined path integral, the uncertainty must be shared between the initial size and the initial momentum, with the uncertainty being of the order of the Hubble length.

In conclusion, let us note that the present implementation of the no-boundary proposal effectively corresponds to a redefinition. Originally, it was formulated as a sum over compact and regular geometries [2]. Here the sum is redefined to be over geometries with an approximately zero initial size and approximately Euclidean initial momentum. This is just fuzzy enough to allow the quantum theory to pick out a regular no-boundary geometry at the saddle point, but the uncertainty is not so large that singular geometries start contributing, too.

We thank Sebastian Bramberger, Job Feldbrugge, and Neil Turok for numerous discussions. We gratefully acknowledge the support of the European Research Council in the form of the ERC Consolidator Grant No. CoG 772295 "Qosmology."

[1] G. Lemaitre, Republication of: The beginning of the world from the point of view of quantum theory, Nature (London) 127, 706 (1931); Gen. Relativ. Gravit. 43, 2929 (2011).

[2] J. B. Hartle and S. W. Hawking, Wave function of the universe, Phys. Rev. D 28, 2960 (1983); Adv. Ser. Astrophys. Cosmol. 3, 174 (1987).

[3] A. Vilenkin, Creation of universes from nothing, Phys. Lett. 117B, 25 (1982).

[4] J. Feldbrugge, J.-L. Lehners, and N. Turok, Lorentzian Quantum cosmology, Phys. Rev. D 95, 103508 (2017).

[5] J. Feldbrugge, J.-L. Lehners, and N. Turok, No Smooth Beginning for Spacetime, Phys. Rev. Lett. 119, 171301 (2017).

[6] J. Feldbrugge, J.-L. Lehners, and N. Turok, No rescue for the no boundary proposal: Pointers to the future of quantum cosmology, Phys. Rev. D 97, 023509 (2018).

[7] A. Di Tucci and J.-L. Lehners, Unstable no-boundary fluctuations from sums over regular metrics, Phys. Rev. D 98, 103506 (2018).

[8] J.-L. Lehners, No smooth beginning for spacetime, Int. J. Mod. Phys. D 28, 1930005 (2019).

[9] J. D. Dorronsoro, J. J. Halliwell, J. B. Hartle, T. Hertog, and O. Janssen, Real no-boundary wave function in Lorentzian quantum cosmology, Phys. Rev. D 96, 043505 (2017).

[10] J. D. Dorronsoro, J. J. Halliwell, J. B. Hartle, T. Hertog, O. Janssen, and Y. Vreys, Damped Perturbations in the No-Boundary State, Phys. Rev. Lett. 121, 081302 (2018).

[11] J. Feldbrugge, J.-L. Lehners, and N. Turok, Inconsistencies of the new no-boundary proposal, Universe 4, 100 (2018).

[12] A. Vilenkin and M. Yamada, Tunneling wave function of the universe, Phys. Rev. D 98, 066003 (2018).

[13] A. Vilenkin and M. Yamada, Tunneling wave function of the universe II: The backreaction problem, Phys. Rev. D 99, 066010 (2019).

[14] J. J. Halliwell, J. B. Hartle, and T. Hertog, What is the noboundary wave function of the universe, Phys. Rev. D 99, 043526 (2019).

[15] S. P. de Alwis, The wave function of the universe and CMB fluctuations, arXiv:1811.12892. 
[16] J. J. Halliwell and J. Louko, Steepest descent contours in the path integral approach to quantum cosmology. 1. The de Sitter minisuperspace model, Phys. Rev. D 39, 2206 (1989).

[17] C. Teitelboim, Quantum mechanics of the gravitational field, Phys. Rev. D 25, 3159 (1982).
[18] J. J. Halliwell, Derivation of the Wheeler-De Witt equation from a path integral for minisuperspace models, Phys. Rev. D 38, 2468 (1988).

[19] J. J. Halliwell and J. B. Hartle, Integration contours for the no boundary wave function of the universe, Phys. Rev. D 41, 1815 (1990). 